C-A/AP/\#204

May 2005

\title{
Towards a Robust Phase Locked Loop Tune Feedback System
}

\section{The continuous measurement of global betatron coupling using a phase locked loop tune measurement system}

\author{
Rhodri Jones, Peter Cameron, Yun Luo
}

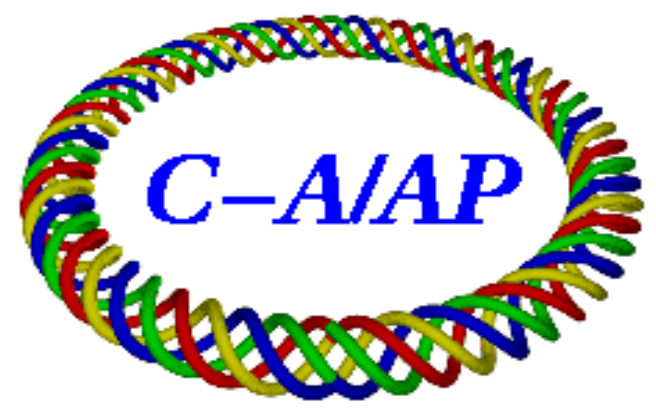

Collider-Accelerator Department Brookhaven National Laboratory

Upton, NY 11973 


\title{
Towards a Robust Phase Locked Loop \\ Tune Feedback System
}

\section{The continuous measurement of global betatron coupling using a phase locked loop tune measurement system}

\author{
Rhodri Jones $^{\dagger}$, Peter Cameron*, Yun Luo* \\ *Brookhaven National Laboratory, Upton, NY 11973, USA \\ ${ }^{\dagger}$ CERN, European Organization for Nuclear Research, CH-1211 Geneva 23, Switzerland
}

\begin{abstract}
Attempts to introduce a reliable tune feedback loop at RHIC have been thwarted by two main problems, namely transition crossing and betatron coupling. The problem of transition crossing is a dynamic range problem, resulting from the increase in the revolution content of the observed signal as the bunch length becomes short and from the fast orbit changes that occur during transition. The dynamic range issue is being addressed by the development of a baseband tune measurement system as part of the US LHC Accelerator Research Program (US-LARP). This paper will focus on the second problem, showing how a phase locked loop tune measurement system can be used to continuously measure global betatron coupling and in so doing allow for robust tune measurement and feedback in the presence of coupling.
\end{abstract}

$22^{\text {nd }}$ April 2005 


\title{
Towards a Robust Phase Locked Loop Tune Feedback System - The continuous measurement of global betatron coupling using a phase locked loop tune measurement system
}

\author{
Rhodri Jones $^{\dagger}$, Peter Cameron ${ }^{*}$, Yun Luo* \\ *Brookhaven National Laboratory, Upton, NY 11973, USA \\ ${ }^{\dagger}$ CERN, European Organization for Nuclear Research, CH-1211 Geneva 23, Switzerland
}

\begin{abstract}
Attempts to introduce a reliable tune feedback loop at RHIC [1] have been thwarted by two main problems, namely transition crossing and betatron coupling. The problem of transition crossing is a dynamic range problem, resulting from the increase in the revolution content of the observed signal as the bunch length becomes short and from the fast orbit changes that occur during transition. The dynamic range issue is being addressed by the development of a baseband tune measurement system [2] as part of the US LHC Accelerator Research Program (US-LARP). This paper will focus on the second problem, showing how a phase locked loop (PLL) tune measurement system can be used to continuously measure global betatron coupling, and in so doing allow for robust tune measurement and feedback in the presence of coupling.
\end{abstract}

\section{Introduction}

There are two main difficulties associated with utilizing a PLL tune measurement and feedback system in the presence of coupling. The first arises from the fact that in a coupled machine the excitation from one plane shows up in the other. A PLL therefore has the possibility to become confused regarding which signal is associated with a given measurement plane. This difficulty exists independent of whether or not tune measurements are fed back through the quadrupoles to control the tunes. If, however, such a signal is now used as input for tune feedback, then the magnet loop will feed back on the wrong plane, causing both the PLL and magnet feedback systems to become unstable.

The second difficulty arises when a tune feedback system tries to maintain the tunes at their 'set' tune values. In the presence of coupling these set, or 'unperturbed', tune values will differ from the measured values by an amount which depends on the coupling amplitude and the distance between the two unperturbed tunes. When the coupling amplitude becomes larger than the difference in the unperturbed tunes, then no amount of quadrupole adjustment can diminish this minimum tune split and restore the tunes to their desired 'set' values.

As a result of these difficulties, a tune feedback loop will only function robustly if coupling has been corrected, while correcting the coupling implies setting up the machine without tune feedback, so making the feedback redundant. The severity of these difficulties became evident during efforts to run RHIC with tune feedback in the polarized proton portion of the 2004 run. The problem with coupling control arises from the need for strong sextupoles in large superconducting accelerators, and the fact that coupling is introduced as a result of vertical orbit changes in these sextupoles. As this obstacle to tune feedback became evident, the need for improved coupling measurement became clear, and the PLL was reconfigured to permit measurement of the projections of both eigenmodes in both planes (see the following section for a discussion of eigenmodes). The excellent quality of the data obtained by this method motivated the development of a proper formalism [3] for its interpretation.

This introduces several possibilities. First, it permits to establish robust PLL tune measurement in the presence of coupling. Second, continuous measurement of the coupling and measured tunes permits realtime calculation of the unperturbed tunes, which can then be provided to the quadrupoles for stable tune feedback. In this way, although the measured tunes may be pushed apart by coupling, the unperturbed tunes will remain constant and hence the feedback loop will be stable. In addition, by keeping the feedback loop stable, the PLL system can be used to measure the coupling during the energy ramp and allow for subsequent coupling correction. Finally, reliable continuous coupling measurement opens the possibility of coupling feedback, in addition to the tune feedback. 


\section{Measurement of Coupling Parameters using a PLL Tune Tracker}

This section will discuss the use of a phase locked loop tune tracker to measure the betatron coupling amplitude and phase.
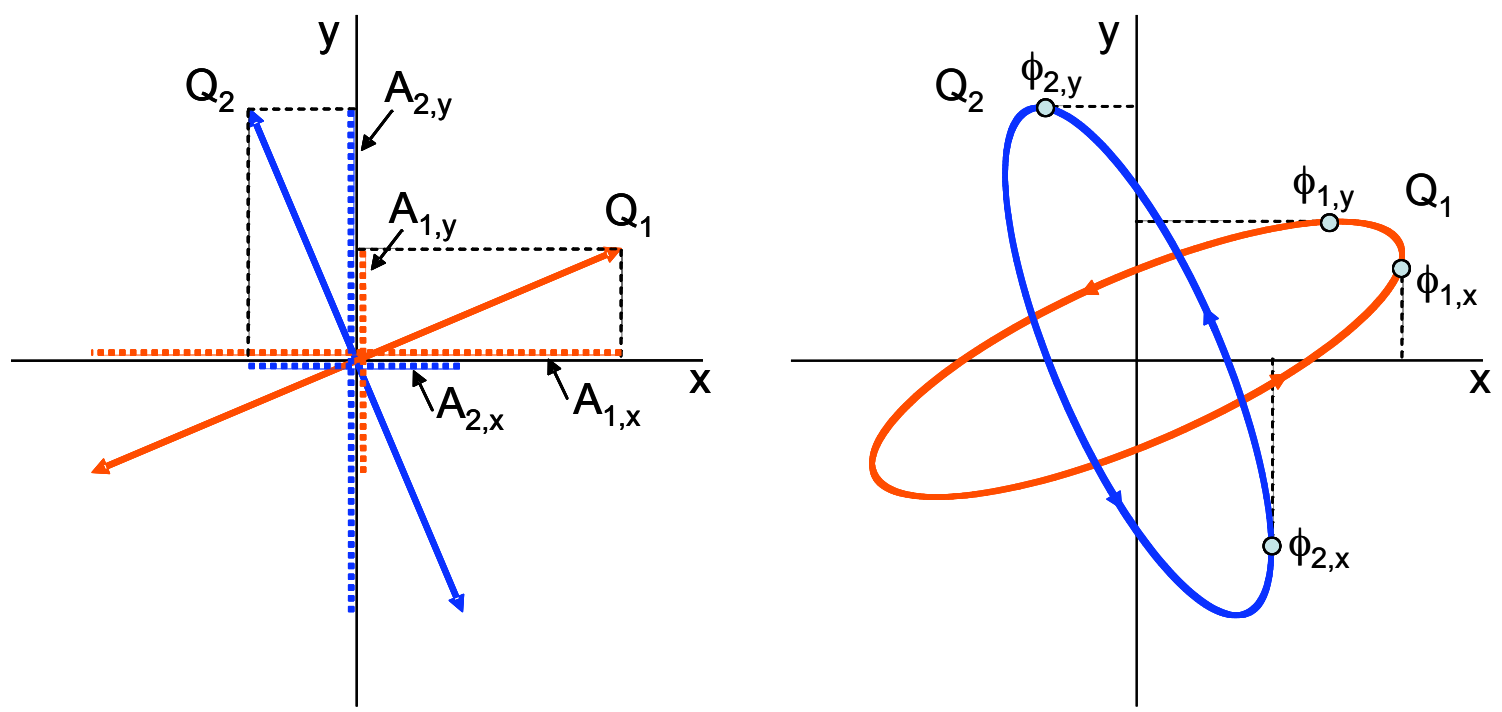

Fig. 1. Schematics showing the two eigenmodes rotated with respect to the horizontal and vertical planes due to coupling. The left hand figure shows the special case where the projections of each mode in each plane are in phase. The right hand side shows the more general case where coupling introduces a phase shift into the eigenmode projections.

\subsection{Equations of Interest}

For a linearly coupled circular accelerator the observed displacement on turn $\mathrm{n}$ in the horizontal (x) and vertical (y) planes are a combination of the projections of two eigenmodes (see [3] and references therein). This is illustrated in Figure 1 and can be expressed as

$$
\left\{\begin{array}{l}
x(n)=A_{1, x} \cos \left(2 \pi Q_{1} n+\phi_{1, x}\right)+A_{2, x} \cos \left(2 \pi Q_{2} n+\phi_{2, x}\right) \\
y(n)=A_{1, y} \cos \left(2 \pi Q_{1} n+\phi_{1, y}\right)+A_{2, y} \cos \left(2 \pi Q_{2} n+\phi_{2, y}\right)
\end{array}\right.
$$

Here it is assumed that Mode 1 is more linked to the horizontal plane, while Mode 2 is more linked to the vertical. The eigenmode frequency of Mode 1 is denoted by $Q_{1}$, while $A_{1, x}$ and $A_{1, y}$ represent the amplitudes of this mode in the horizontal and vertical plane respectively. Similarly $\phi_{1, \mathrm{x}}$ and $\phi_{1, \mathrm{y}}$ represent the phases of this mode in the horizontal and vertical plane respectively. The same notation applies for the frequency, amplitudes, and phases of Mode 2.

Using Hamiltonian perturbation theory in the absence of intentionally strong local couplers, it can be shown (see [3] and references therein) that the general expression for coupled betatron oscillations in the $\mathrm{x}$ and $\mathrm{y}$ plane along the reference trajectory can be written as

$$
\left\{\begin{array}{l}
x(s)=\sqrt{2 \beta_{x}}\left\{a \cos \left[\Psi_{x}+(v-\Delta / 2) \varphi-\chi / 2\right]+b \cos \left[\Psi_{x}-(v+\Delta / 2) \varphi-\chi / 2\right]\right\} \\
y(s)=\sqrt{2 \beta_{y}}\left\{c \cos \left[\Psi_{y}+(v+\Delta / 2) \varphi+\chi / 2\right]+d \cos \left[\Psi_{y}-(v-\Delta / 2) \varphi+\chi / 2\right]\right\}
\end{array}\right.
$$

where

$$
\frac{c}{a}=-\frac{b}{d}=\frac{\left|C^{-}\right|}{2 v+\Delta} \quad ; \quad v=\frac{1}{2} \sqrt{\Delta^{2}+\left|C^{-}\right|^{2}} \quad ; \quad \varphi=\frac{2 \pi s}{L}
$$


Here $\mathrm{s}$ is the distance along the reference trajectory and $\mathrm{L}$ is the circumference of the accelerator. $\Psi_{\mathrm{x}}$ and $\Psi_{\mathrm{y}}$ are the unperturbed horizontal and vertical angular frequencies, $\Delta$ is the difference between the fractional part of the unperturbed tunes $\left(\Delta=\mathrm{Q}_{\mathrm{x}, 0}-\mathrm{Q}_{\mathrm{y}, 0}-\mathrm{p}\right.$, with $\mathrm{p}$ an integer), and the complex coupling coefficient $\mathrm{C}^{-}$is defined as

$$
C^{-}=\left|C^{-}\right| e^{i \chi}
$$

When considering measurements taken at a single location on a turn-by-turn basis, Eqs. (2) can be rewritten as

$$
\left\{\begin{array}{l}
x(n)=\sqrt{2 \beta_{x}}\left\{a \cos \left[2 \pi\left(Q_{x, 0}-\frac{1}{2} \Delta+v\right) n-\chi / 2\right]+b \cos \left[2 \pi\left(Q_{y, 0}+\frac{1}{2} \Delta-v\right) n-( \pm \pi / 2+\chi / 2)\right]\right\} \\
y(n)=\sqrt{2 \beta_{y}}\left\{c \cos \left[2 \pi\left(Q_{x, 0}-\frac{1}{2} \Delta+v\right) n+\chi / 2\right]+d \cos \left[2 \pi\left(Q_{y, 0}+\frac{1}{2} \Delta-v\right) n+( \pm \pi / 2+\chi / 2)\right]\right\}
\end{array}\right.
$$

Comparing Eq.(3) with Eq.(1), the following variables can be defined [3]:

$$
\begin{aligned}
& \left\{\begin{array}{l}
r_{1}=\frac{A_{1, y}}{A_{1, x}}=\sqrt{\frac{\beta_{y}}{\beta_{x}}} \cdot \frac{\left|C^{-}\right|}{2 v+\Delta} \\
r_{2}=\frac{A_{2, x}}{A_{2, y}}=\sqrt{\frac{\beta_{x}}{\beta_{y}}} \cdot \frac{\left|C^{-}\right|}{2 v+\Delta}
\end{array}\right. \\
& \left\{\begin{array}{l}
\Delta \phi_{1}=\phi_{1, y}-\phi_{1, x}=\chi \\
\Delta \phi_{2}=\phi_{2, x}-\phi_{2, y}= \pm \pi-\chi
\end{array}\right.
\end{aligned}
$$

It is also possible to write the following relations for the eigenmode frequencies, $\mathrm{Q}_{1}$ and $\mathrm{Q}_{2}$

$$
\begin{aligned}
& \left\{\begin{array}{l}
Q_{1}=Q_{x, 0}-\frac{1}{2} \Delta+\frac{1}{2} \sqrt{\Delta^{2}+\left|C^{-}\right|^{2}} \\
Q_{2}=Q_{y, 0}+\frac{1}{2} \Delta-\frac{1}{2} \sqrt{\Delta^{2}+\left|C^{-}\right|^{2}}
\end{array}\right. \\
& \left|Q_{1}-Q_{2}\right|=\sqrt{\Delta^{2}+\left|C^{-}\right|^{2}}
\end{aligned}
$$

Solving for $\Delta$ and $\left|\mathrm{C}^{-}\right|$using Eqs.(4) and (7) one obtains

$$
\left|C^{-}\right|=\frac{2 r_{1} \sqrt{\frac{\beta_{y}}{\beta_{x}}}\left|Q_{1}-Q_{2}\right|}{\left(\frac{\beta_{y}}{\beta_{x}}+r_{1}^{2}\right)} \quad, \quad \Delta=\frac{\left|Q_{1}-Q_{2}\right|\left(\frac{\beta_{y}}{\beta_{x}}-r_{1}^{2}\right)}{\left(\frac{\beta_{y}}{\beta_{x}}+r_{1}^{2}\right)}
$$

From Eqs.(4) it can be seen that $\mathrm{r}_{1}=\beta_{\mathrm{y}} / \beta_{\mathrm{x}} \mathrm{r}_{2}$. Substituting into Eqs.(8) one therefore obtains the expressions

$$
\left|C^{-}\right|=\frac{2 \sqrt{r_{1} r_{2}}\left|Q_{1}-Q_{2}\right|}{\left(1+r_{1} r_{2}\right)} \quad, \quad \Delta=\frac{\left|Q_{1}-Q_{2}\right|\left(1-r_{1} r_{2}\right)}{\left(1+r_{1} r_{2}\right)}
$$

which are independent of the beta functions at the observation location. 


\subsection{Configuring a PLL tune tracker to measure betatron coupling parameters}

In the case of a decoupled machine a PLL tune tracker will simply follow the horizontal and vertical unperturbed tunes. Once coupling is introduced the plane of decoupled oscillations is rotated with respect to the horizontal and vertical planes (see Fig. 1) and the PLL will now track the frequency of one of the two eigenmodes. When the difference $\Delta$ between the unperturbed tunes is much greater than the minimum tune split $\left|\mathrm{C}^{-}\right|$, the eigenmodes are only slightly rotated with respect to the observation planes. In this case one of the eigenmodes will have a much larger amplitude of oscillation than the other for a given plane and the PLL will naturally lock onto this favoured eigenmode (here assumed to be eigenmode 1 for the horizontal PLL and eigenmode 2 for the vertical). When $\Delta$ approaches zero, the eigenmodes will rotate by $45^{\circ}$ and the PLL will see similar amplitudes for both eigenmode frequencies. Depending on the frequency difference between the two eigenmodes, given by $\left|\mathrm{C}^{-}\right|$in this case, and the relative beta functions and excitation amplitudes associated with the eigenmode projections, the PLL may become unstable and jump from one eigenmode frequency to the other.

Further reducing $\Delta$ to negative values results in the crossing of the unperturbed tunes and causes the eigenmodes to rotate by more than $45^{\circ}$. Eigenmode 1 will now have more amplitude in the vertical plane while eigenmode 2 will have more amplitude in the horizontal. If $\left|\mathrm{C}^{-}\right|$is sufficiently large to give a reasonable frequency difference between the two eigenmode frequencies, then the PLL may continue to track the same eigenmode even though its amplitude in the plane of observation is now lower than that of the other eigenmode. Obviously in such cases the PLL can become unstable and could lose lock altogether.

What is clear from the above discussion is that the PLL is an eigentune tracker. By reconfiguring the PLL as shown in Figure 2 it can therefore provide all the necessary information to measure coupling. In the classical PLL tune tracker implementation, the horizontal plane is configured to track one eigenmode (assumed to be eigenmode 1), while the vertical tracks the other (eigenmode 2). If, instead, the vertical detection frequency is forced to be the same as that of the horizontal, then the vertical acquisition chain will observe the projection of eigenmode 1 in the vertical plane. By duplicating the number of channels the same principle can be applied to eigenmode 2 .

The amplitude and phase measured by each of these channels are then the same amplitudes and phases described in Eq. (1). Since the PLL kicker is the same for a given eigenmode, its gain and the beta-function at its location do not have to be taken into account when computing the ratios $r_{1} \& r_{2}$. This is not true for the receiver gains, which can be different for each channel.

Eqs. (4) and (5) can therefore be rewritten as

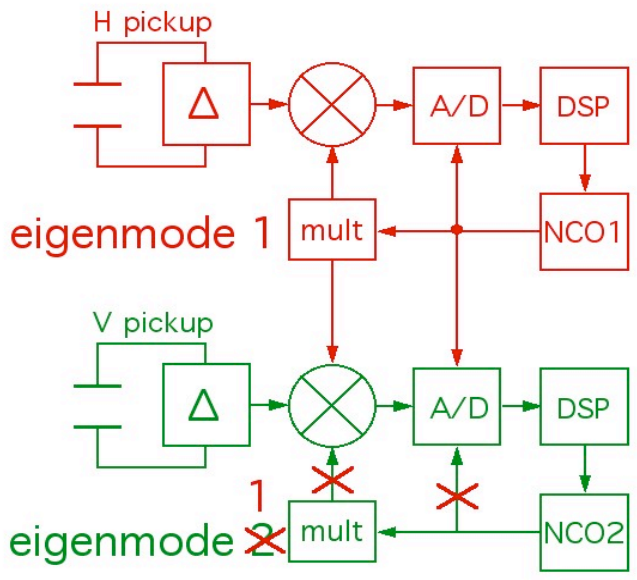

Fig. 2. Schematic representation of the RHIC PLL system as modified for coupling measurement

$$
\left\{\begin{array}{l}
r_{1}=\frac{G_{x} A_{1, y}}{G_{y} A_{1, x}}=\sqrt{\frac{\beta_{y}}{\beta_{x}}} \cdot \frac{\left|C^{-}\right|}{2 v+\Delta} \\
r_{2}=\frac{G_{y} A_{2, x}}{G_{x} A_{2, y}}=\sqrt{\frac{\beta_{x}}{\beta_{y}}} \cdot \frac{\left|C^{-}\right|}{2 v+\Delta}
\end{array} \quad, \quad\left\{\begin{array}{l}
\Delta \phi_{1}=\phi_{1, y}-\phi_{1, x}=\chi \\
\Delta \phi_{2}=\phi_{2, x}-\phi_{2, y}= \pm \pi-\chi
\end{array}\right.\right.
$$

The eigentunes $\mathrm{Q}_{1}$ and $\mathrm{Q}_{2}$ together with the 4 parameters of Eqs. (10) constitute a complete set of global coupling observables [3].

When the PLL is locked to first order $\phi_{1, \mathrm{x}}=\phi_{2, \mathrm{y}}=0$ and hence the coupling phase, $\chi$, is simply given by $\phi_{1, y}\left(\right.$ or $\left.\pm \pi-\phi_{2, y}\right)$. The coupling amplitude, $\left|\mathrm{C}^{-}\right|$, and unperturbed tune difference, $\Delta$, can be obtained using Eqs. (9). In addition the unperturbed tune values $\mathrm{Q}_{\mathrm{x}, 0}$ and $\mathrm{Q}_{\mathrm{y}, 0}$ can be calculated using Eqs. (6). 


\section{Results of Coupling Measurements using a PLL Tune Tracker at RHIC}

To perform continuous coupling measurements during the acceleration ramp, the RHIC PLL tune tracker was configured as shown in Fig. 2. Since the measurement required doubling the number of acquisition channels it could only be performed for one ring. The yellow ring PLL was therefore used in its standard configuration to acquire eigenmode 1 (horizontal) and eigenmode 2 (vertical), while the blue PLL system was used to acquire the projection of yellow eigenmode 1 in the vertical and that of yellow eigenmode 2 in the horizontal.

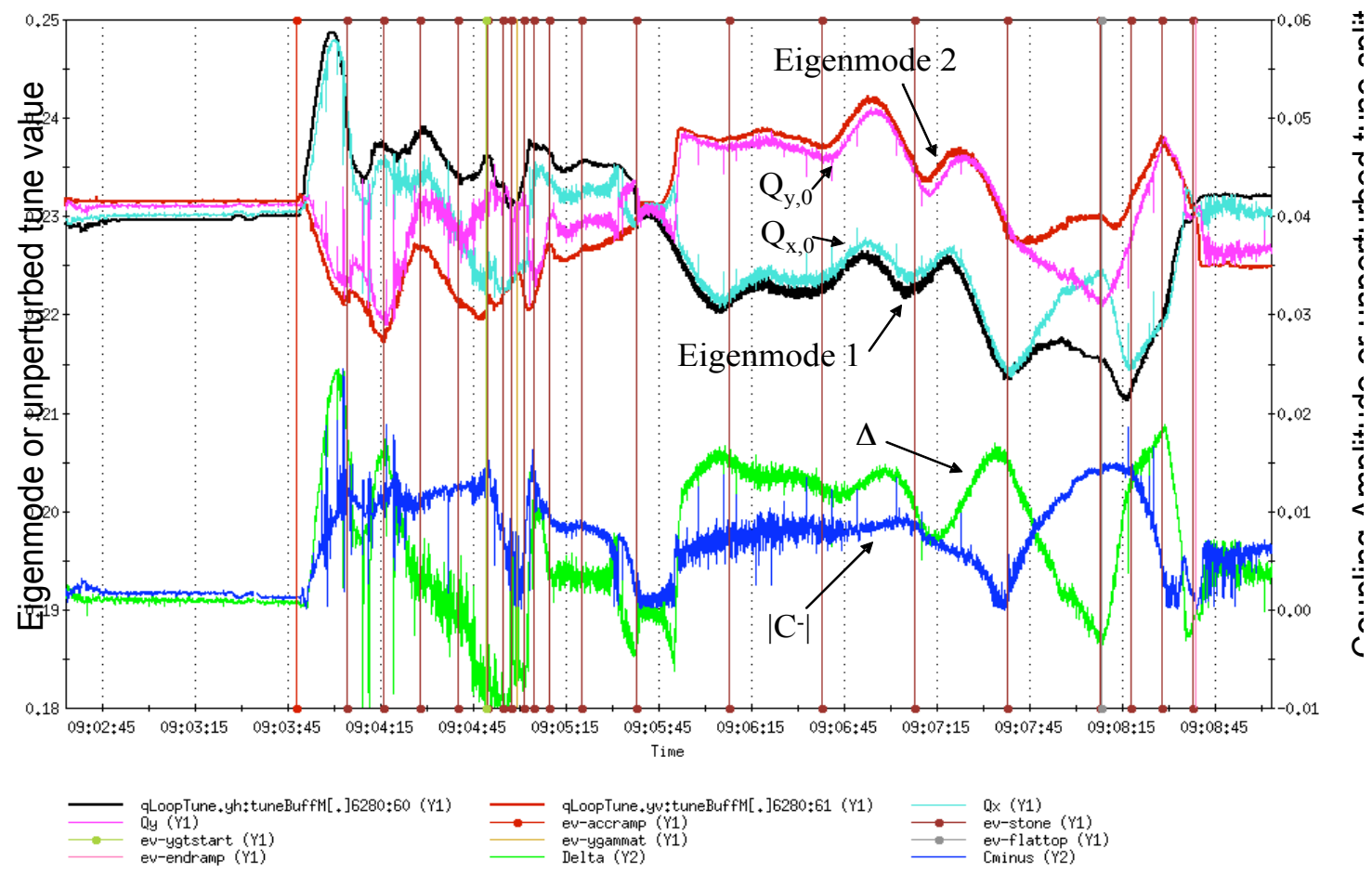

Fig. 3. Continuous coupling amplitude measurement using the PLL tune tracker during a RHIC ramp.

Figure 3 shows the results of one such measurement taken during $\mathrm{Cu}$ run number 6280 . The coupling is seen to be well adjusted during injection, but becomes large near transition and again towards the end of the ramp. When considering only the usual PLL tune data (eigenmodes 1 and 2) the tune would seem to be well adjusted during the early part of the ramp. Looking at the unperturbed tunes $\left(\mathrm{Q}_{\mathrm{x}, 0}\right.$ and $\left.\mathrm{Q}_{\mathrm{y}, 0}\right)$ as calculated from $\left|\mathrm{C}^{-}\right|$and $\Delta$ using Eq. (6), one can clearly see that this is not the case. The unpertubed tunes actually cross during this time, something which was confirmed by the kicked tune measurement system (ARTUS) from a similar, earlier ramp (the kicked tune system was left off during ramp 6280 to minimize beam loss due to emittance blowup). This is a good example of where the PLL will continue to track a given eigenmode, even though its major projection is now in the other plane. Incidentally, this leads to a negative $\Delta$ at these locations, since the amplitude ratios $r_{1}$ and $r_{2}$ become larger than 1 due to the fact that the PLL is now tracking the eigenmode with the smallest projection amplitude in both planes.

The evolution of the phase differences, $\Delta \phi_{1}$ and $\Delta \phi_{2}$, during $\mathrm{Cu}$ ramp 6280 are shown in Fig. 4. Knowledge of the amplitude and phase of the complex coupling coefficient should allow global coupling to be corrected using two orthogonal skew quadrupole families. From Eqs. (10) the difference between the two measured phase differences $\Delta \phi_{1}$ and $\Delta \phi_{2}$ is expected to be $\pm \pi$. This is clearly not the case in Fig. 5, where the difference seems to take values close to the discrete values $-2 \pi, \pi, 0,+2 \pi$. The origin of this discrepancy is yet to be understood and either comes from the way in which the PLL locks to the unfavourable eigenmode or from spurious phase introduced by local coupling. 


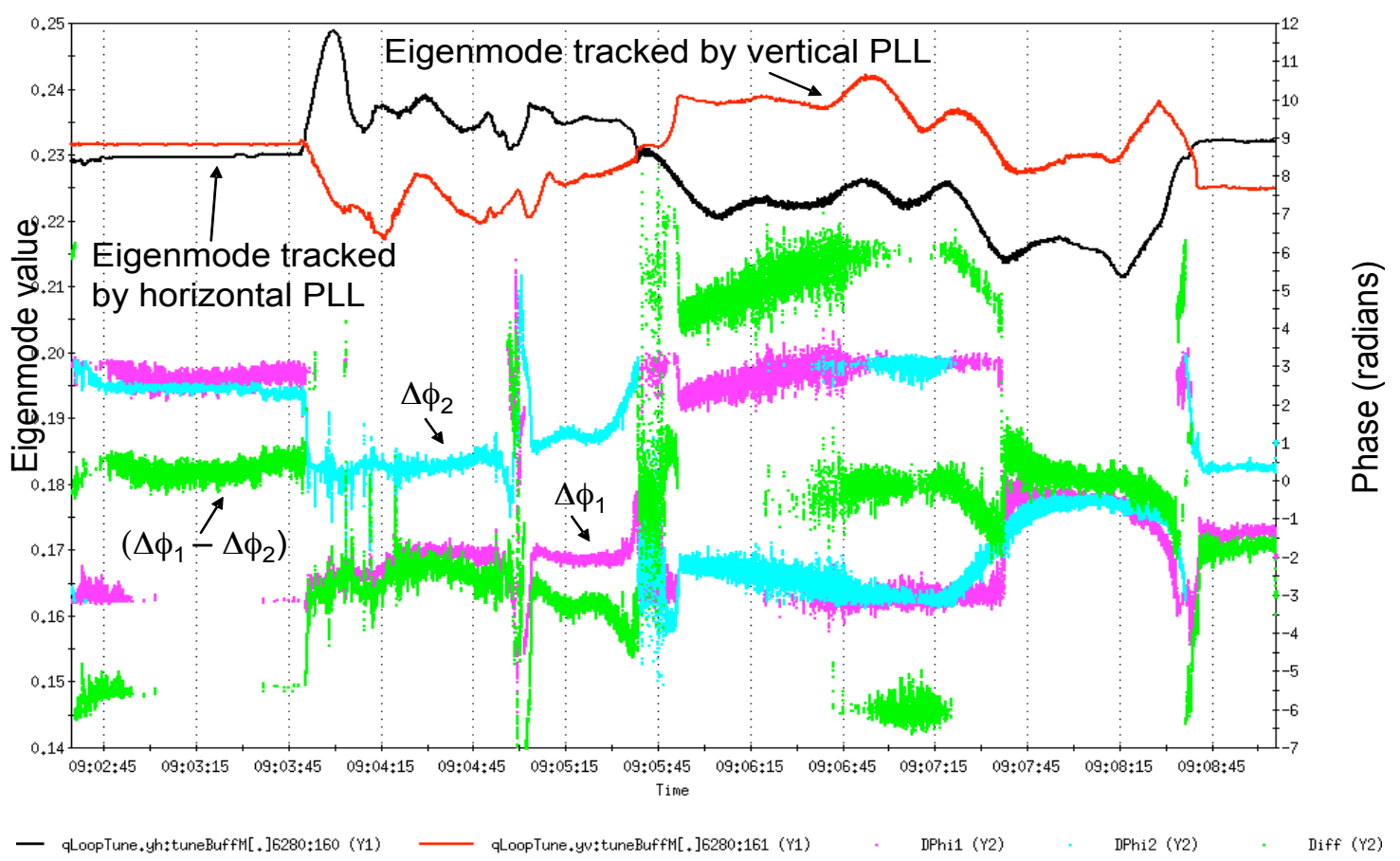

Fig. 4. Continuous measurement of the coupling phase $\chi$ using $\Delta \phi_{1}$ and $\Delta \phi_{2}$.

The PLL was also configured to track all four eigenmode projections of a single beam during the proton run, when a working point favorable to polarization was selected above the half-integer, unlike the working point below the half-integer that was used in the Copper run and shown in the previous figures.

The lower image of Fig. 5 shows the measured eigentunes $Q_{I}$ (blue continuous trace) and $Q_{\text {II }}$ (green continuous trace), as well as the same eigentunes measured at 2 second intervals by the kicked tune system (purple and grey dots). The upper image shows the coupling coefficient (red) and uncoupled tune split (black), calculated as described above. From these, the fractional portion of the unperturbed 'set' tunes $\mathrm{Q}_{\mathrm{x}, 0}$ (black) and $\mathrm{Q}_{\mathrm{y}, 0}$ (red) are calculated and displayed in the lower image.

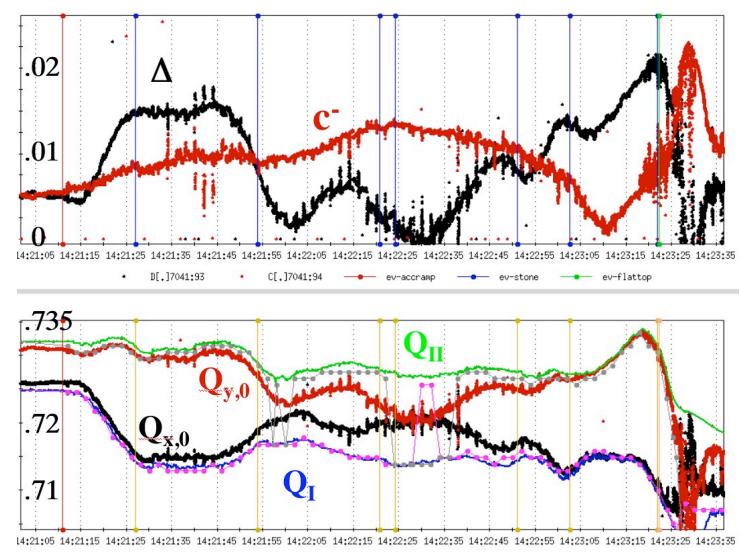

Fig. 5. Tune and coupling data from ramp 7041

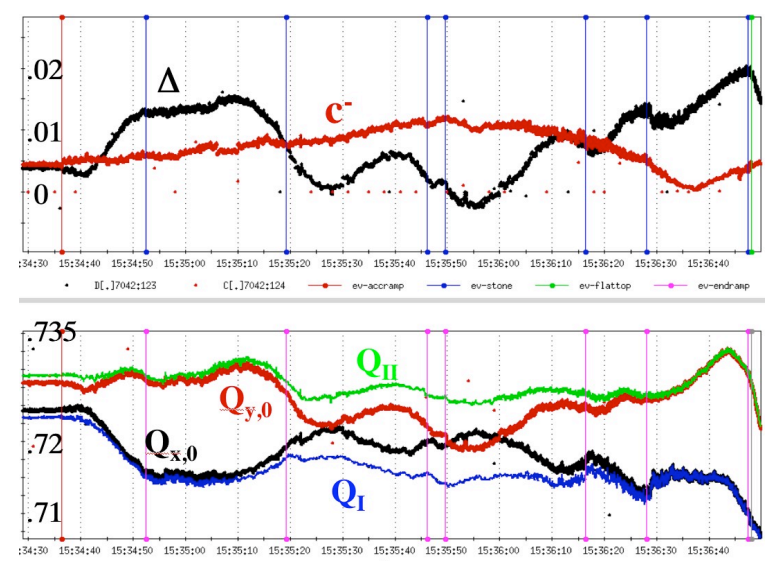

Fig. 6. Tune and coupling data from ramp 7042

It can be seen that for the first $\sim 40$ seconds of the ramp the machine is reasonably well decoupled, and the tune split is due almost entirely to the uncoupled tune split. For the next minute or so the coupling becomes large, at times so large that the tune split is due entirely to the coupling. And for the last $\sim 30$ seconds of data the machine is again reasonably well decoupled. During the interval in which the coupling 
is large, the PLL continues to track the correct eigenmodes. The kicked tune measurement, however, clearly becomes confused regarding which plane corresponds to which eigenmode. It is also interesting to note that while the PLL tune measurement is apparently unperturbed by the firing of the tune kicker every two seconds, the coupling measurement is strongly affected by the resulting coherent motion at some (but not all!) times. Strong dependence of coupling on orbit has been independently observed in RHIC.

Data taken in the ramp immediately after that shown in Figure 5 is presented in Figure 6. This data demonstrates that the measurement is repeatable. The kicked tune system did not operate for this ramp, and the absence of the resulting perturbation to the coupling is quite evident.

\section{Making a PLL Tune Tracker Robust in the Presence of Betatron Coupling}

By tracking a single eigenmode in each plane, as is typically done by most PLL tune measurement systems, it is very easy for the PLL to become confused when the unperturbed tunes are close together and the coupling amplitude becomes large. This can lead to the PLL in both planes tracking the same eigenmode, or with one of the planes losing lock altogether.

The addition of the information related to the projection of the excitation in the other plane allows the PLL to know the state of its current eigenmode with respect to that being tracked by the other plane. This means that if the unperturbed tunes cross, then the horizontal and vertical PLLs can be forced to change from one eigenmode to the other. In this way they keep track of the eigenmode with the largest amplitude projection in their respective planes, reducing the chance of losing lock should the coupling amplitude decrease.

As was shown in the previous section, if a robust tune tracker can be successfully implemented during the ramp, then coupling is automatically measured. Taking this one step further one could even imagine performing direct coupling feedback using the parameters measured by the PLL.

\section{Making PLL Tune Feedback Robust in the Presence of Betatron Coupling}

Section 3 has shown how it is possible to configure a PLL tune tracker to continuously measure the coupling parameters. By knowing $\left|\mathrm{C}^{-}\right|$and $\Delta$ and the two eigenmode frequencies $\mathrm{Q}_{1}$ and $\mathrm{Q}_{2}$ it is possible to determine the unperturbed tunes, $\mathrm{Q}_{\mathrm{x}, 0}$ and $\mathrm{Q}_{\mathrm{y}, 0}$, that would be measured in the absence of betatron coupling. The nice thing about the unperturbed tunes is that they remain constant for any value of the coupling amplitude. This means that these values could be used in a tune feedback loop without giving rise to the problems encountered when feeding back on the eigenmode frequencies.

In practice this would imply that the set tunes requested by the tune feedback loop would be the unperturbed tunes rather than the actual oscillation frequencies (eigenmodes) undertaken by the beam. The feedback loop would therefore be stable in the presence of coupling, but would not prevent the beam from oscillating on or near resonances were coupling to become large. By performing tune feedback in this manner, however, the problems of coupling and tune correction become two separate issues rather than being interdependent.

\section{Conclusions}

Results from RHIC have shown that continuous betatron coupling measurements can be obtained throughout the acceleration ramp by appropriately configuring a PLL tune tracker. Not only does this provide the coupling amplitude, but also the coupling phase, both of which are required for successful coupling correction. The ability to measure the coupling parameters gives a PLL tune tracker two added advantages: the ability to track tunes in the presence of betatron coupling, and the ability to provide the necessary quantities for an 'unperturbed' tune feedback system in the presence of betatron coupling. All of this greatly enhances the power of a PLL tune measurement system, and points the way to a robust tune feedback system for RHIC and future hadron machines.

So far the effect of local coupling on the measurements has not been studied, but future beam experiments will attempt to verify that the observed quantities do indeed allow for global betatron coupling correction or eventual feedback. 


\section{Acknowledgement}

The authors acknowledge well-considered guidance from Steve Peggs, as well as many helpful conversations.

\section{References}

[1] P. Cameron et al, "RHIC Third Generation PLL Tune Measurement System”, PAC 2003. http://epaper.kek.jp/p03/PAPERS/ROAB009.PDF

[2] M. Gasior and R. Jones, "High Sensitivity Tune Measurement by Direct Diode Detection", DIPAC 2005.

http://dipac2005.web.cern.ch/dipac2005/default.htm

[3] Y. Luo, P. Cameron, S. Peggs, D. Trbojevic, "Possible phase loop for the global betatron decoupling", RHIC-AP-174.

http://www.cadops.bnl.gov/AP/ap notes/cad ap index.html 